\title{
Energy Optimization of a High Consistency Refiner Process
}

\author{
Patrick Höhn* Wolfgang Birk** \\ * Control Engineering Group, Department of Computer Science, \\ Electrical and Space Engineering, Lulea University of Technology, \\ SE-97187 Luleå, Sweden (e-mail: patrick.hohn@ltu.se). \\ ** Control Engineering Group, Department of Computer Science, \\ Electrical and Space Engineering, Luleå University of Technology,
} SE-971 87 Luleå, Sweden

Keywords: High consisteny refining, energy optimization, Gurley number, entropy model, explicit solution

\section{INTRODUCTION}

A concept for energy optimization of a high consistency (HC) refining processes in the pulp and paper industry is presented. High consistency refining is an important stage in the production of Kraft paper as it is required to obtain the desired paper properties Brännvall (2004). Refiners as discussed here consist of one rotating disc and one static disc. The pulp is pressed through the narrow gap between both and processed.

Two kinds of models are used for the optimization - one describing the behavior inside the refining zone with an entropy based approach and the other is an empirical model connecting the specific energy to a pulp quality parameter given by the Gurley number. The latter model is also denoted as the quality parameter model.

This optimization uses the specific energy as manipulated variable which is defined as energy consumption per the mass flow rate in the refiner. Optimized variables that should stay close to their desired values are the outlet consistency and the Gurley number. The outlet consistency is defined as the solid fraction of the pulp after the refiner. Moreover, the Gurley number is a measure for the air resistance of the produced sack paper and is defined by the time required by $100 \mathrm{ml}$ of air to flow through a test sample of paper with an area of $6.45 \mathrm{~cm}^{2}$ Its model was provided by industrial partners based on experiments performed by Fridén et al. (2006). The outlet consistency is determined by using a refiner model which is based on the entropy model by Eriksson (2005). However, the model is extended for the current optimization. Under certain assumptions a predetermined temperature distribution in the refiner is used to calculate various thermodynamics properties like enthalpy, entropy, saturation pressure of steam.

The optimization permits to adjust the inlet consistency to a fixed value and getting the corresponding solution. While keeping the energy consumption as low as possible, it is still attempted to keep outlet consistency and Gurley number close to its desired value. An important parameter in the model is the temperature profile since it has a large influence on the steam creation in the refining zone as

\footnotetext{
* Funding provided by the European Structural Funds is hereby gratefully acknowledged.
}

presented by Höhn and Birk (2013). Here the temperature profile is generated by a cubic approximation between the inlet, maximum and outlet temperature for different radial positions. Discretizing the refining zone over the radius yields four algebraic equations for the mass flow rates of steam, water and fibers in each radial element.

\section{OPTIMIZATION PROBLEM}

A convex optimization procedure is used which involves a cost function with quadratic terms and takes squares of deviations from the desired quality parameters to minimize the specific energy. Its cost function is given below:

$$
\begin{aligned}
J= & (S E)^{2}+\bar{W}_{1}\left(\frac{C\left(r_{1}\right)}{1-C\left(r_{1}\right) K_{1} S E}-C_{S P}\right)^{2} \\
& +\bar{W}_{2}\left(K_{2}\left(S E-S E_{0}\right)+G u_{0}-G u_{S P}\right)^{2}
\end{aligned}
$$

The optimization problem has been solved using both analytical and numerical approaches. Analytically the solution can be obtained by using the first derivate of the cost function and equaling it to zero. Respective expressions can be found below:

$$
\frac{d J}{d S E}=a S E^{4}+b S E^{3}+c S E^{2}+d S E+e,
$$

where the coefficients can be written as expressions of $C\left(r_{1}\right), C_{S P}, \Delta G u, K_{1}, K_{2}, \bar{W}_{1}$ and $\bar{W}_{2}$ :

$$
\begin{aligned}
a & =-2 C\left(r_{1}\right)^{3} K_{1}^{3}\left(K_{2}^{2} \bar{W}_{2}+1\right) \\
b & =2 C\left(r_{1}\right)^{2} K_{1}^{2}\left(C\left(r_{1}\right) K_{1} K_{2}^{2} S E_{0} \bar{W}_{2}\right. \\
& \left.+3 K_{2}^{2} \bar{W}_{2}-C\left(r_{1}\right) \Delta G u K_{1} K_{2} \bar{W}_{2}+3\right) \\
c & =-6 C\left(r_{1}\right) K_{1}\left(C\left(r_{1}\right) K_{1} K_{2}^{2} S E_{0} \bar{W}_{2}\right. \\
& \left.+K_{2}^{2} \bar{W}_{2}-C\left(r_{1}\right) \Delta G u K_{1} K_{2} \bar{W}_{2}+1\right) \\
d & =2\left(3 C\left(r_{1}\right) K_{1} K_{2}^{2} S E_{0} \bar{W}_{2}+K_{2}^{2} \bar{W}_{2}\right. \\
& -3 C\left(r_{1}\right) \Delta G u K_{1} K_{2} \bar{W}_{2} \\
& \left.+C\left(r_{1}\right)^{3} C_{S P} K_{1}^{2} \bar{W}_{1}+1\right) \\
e & =-2\left(K_{2}^{2} S E_{0} \bar{W}_{2}-\Delta G u K_{2} \bar{W}_{2}\right. \\
& \left.+C\left(r_{1}\right)^{2} C_{S P} K_{1} \bar{W}_{1}-C\left(r_{1}\right)^{3} K_{1} \bar{W}_{1}\right)
\end{aligned}
$$




\section{EXPLICIT AND NUMERICAL SOLUTION}

The complete solution can then be determined by using the solution equation for a quartic function:

$$
\begin{aligned}
S E_{1,2} & =-\frac{b}{4 a}-S \pm \frac{1}{2} \sqrt{-4 S^{2}-2 p+\frac{q}{S}} \\
S E_{3,4} & =-\frac{b}{4 a}+S \pm \frac{1}{2} \sqrt{-4 S^{2}-2 p-\frac{q}{S}} \\
p & =\frac{8 a c-3 b^{2}}{8 a^{2}} \\
q & =\frac{b^{3}-4 a b c+8 a^{2} d}{8 a^{3}} \\
S & =\frac{1}{2} \sqrt{-\frac{2}{3} p+\frac{1}{3 a}\left(Q+\frac{\Delta_{0}}{Q}\right)} \\
Q & =\sqrt[3]{\frac{\Delta_{1}+\sqrt{\Delta_{1}^{2}-4 \Delta_{0}^{3}}}{2}} \\
\Delta_{0} & =c^{2}-3 b d+12 a e \\
\Delta_{1} & =2 c^{3}-9 b c d+27 b^{2} e+27 a d^{2}-72 a c e
\end{aligned}
$$

Further analysis of the solution can be done using a gradient based approach. Among the four solutions only one gives a physical solution for specific energy, i.e. the other three solutions lie either outside a reasonable range or render in complex numbers. Evaluation of the solution shows that the used parameters in the solution have a higher influence compared to the introduced weighting factors. Among the two weights the value of $W_{1}$ has a very small impact on the solution compared to $W_{2}$. The explicit solution is compared for distinct points to a numerical optimization using a global search algorithm and interior point method. Details of the utilized optimization algorithms can be found in publications by Glover (1998), Byrd et al. (1999) and Byrd et al. (2000).

\section{CONCLUSION}

Optimization results from both approaches are compared and found to match closely with each other. It resulted in a reduction of energy from currently applied $250 \mathrm{kWh}$ to $247 \mathrm{kWh}$. Moreover, it is found that the relative deviation in Gurley number is half of the relative deviation of SE. Hence there exists a potential for a reduction of energy consumption. Additionally, the explicit solution provides a simple and fast solution to determine the optimal specific energy for selected values of outlet consistency and Gurley number.

\section{NOTATION}

\section{Symbol Explanation}

$S E \quad$ specific energy

(work per tonne applied to pulp)

$S E_{0} \quad$ specific energy at operating point

$C \quad$ consistency (solid mass fraction of pulp)

$C_{S P} \quad$ desired consistency for optimization

$C_{\text {out }} \quad$ consistency at outlet

(solid mass fraction of pulp)

$r_{1} \quad$ inner radius of refiner segments

$r_{2} \quad$ outer radius of refiner segments

\section{Symbol}

Gu

$G u_{S P}$

$G u_{0}$

$\Delta G u$

$a, b, c, d, e$

$\bar{W}_{1}$

$\bar{W}_{2}$

$K_{1}$

$K_{2}$
Explanation

Gurley (Pulp Quality Parameter)

desired Gurley (Pulp Quality Parameter)

for optimization

operating point for the pulp quality

model

difference between operating point and desired value for the pulp quality model

coefficients in the explicit solution

weight for consistency term

weight for gurley term

constant for temperature profile

constant from the pulp quality model

\section{ACKNOWLEDGEMENTS}

The support by the SCOPE consortium and Mondi Dynäs $\mathrm{AB}$ is warmly appreciated.

\section{REFERENCES}

Brännvall, E. (2004). Pulp characterization. In The Ljungberg textbook. Pulp technology 2004, Trita-FPT-Report, 1652-2443 ; 2004:33, 10-12. Stockholm. 00000.

Byrd, R.H., Gilbert, J.C., and Nocedal, J. (2000). A trust region method based on interior point techniques for nonlinear programming. Mathematical Programming, 89(1), 149-185. 00474.

Byrd, R.H., Hribar, M.E., and Nocedal, J. (1999). An interior point algorithm for large-scale nonlinear programming. SIAM Journal on Optimization, 9(4), 877-900. 00579 .

Eriksson, K. (2005). An Entropy-based Modeling Approach to Internally Interconnected TMP Refining Processes. Licentiate, Chalmers University of Technology, Göteborg, Sweden.

Fridén, H., Näslund, H., Stattin, A., and Löfgren, G. (2006). Online decision support and optimization for kraft paper production. In Proceedings of the Control Systems 2006, 313. Kopio Niini - Painoporssi,, Tampere, Finland. 00000.

Glover, F. (1998). A template for scatter search and path relinking. In Artificial evolution, 1-51. 00595.

Höhn, P. and Birk, W. (2013). Sensitivity analysis of models for high consistency refining process. In Proceedings of the 2013 IEEE Multi-conference on Systems and Control. Hyderabad, India. 